\title{
Common Challenges in Simulating the Fluid Dynamics of Electrowetting-On-Dielectrics Driven Devices
}

\author{
Andreas Tröls, Bernhard Jakoby \\ Institute for Microelectronics and Microsensors, Johannes Kepler University, Linz-4040, Austria, \\ Corresponding Authors E-Mail: andreas.troels@jku.at
}

\begin{abstract}
Simulating fluid driven devices is a challenging task in terms of verifying the results provided by the solution algorithm. We particularly consider solvers based on the finite element method (FEM) provided by the commercial software $\mathrm{COMSOL}^{\mathrm{TM}}$ Multiphysics. The three-dimensional Navier-Stokes equations are highly dependent on the initial conditions and can only be solved numerically for particularly considered applications. Choosing a suitable solution algorithm is one of the main tasks when working in the field of numerical fluid dynamics. For so-called Electrowetting-On-Dielectric (EWOD) driven systems, the main focus lies on interface tracking algorithms, like the well-known LevelSet or PhaseField method. The interfacial shape of a fluid in contact with another fluid or gas is governed by the equilibrium of their surface tensions. EWOD is a surface tension driven effect and so the correct reconstruction of the fluid interface is crucial for reliable FEM simulation results. Also the quality of the numerical mesh and its treatment during a movement of the fluid interface through the calculation area is essential. This work reviews the sensitivity of EWOD FEM simulation results on the chosen solver and gives simulation examples as well as a recommendation for the most suitable algorithm and numerical mesh settings. It also shows an example of the simulation results for scaling the fluid channel of an EWOD driven device, which was investigated recently, and the associated scaling limits obtained.
\end{abstract}

Key words: Electrowetting, Microfluidics, Finite Element Simulation, CFD, LevelSet, PhaseField;

\section{Introduction}

Common challenges in the field of numerical fluid dynamics are the choice of the correct algorithm, solver, mesh representation and quality, and of course choosing the correct boundary and initial conditions for a specific multiphase flow problem. For instance, a more accurate simulation with a larger number of considered variables and physical effects is always a tradeoff against the rising computational effort with every extra variable or mesh element, which has to be calculated during a time dependent CFD-FEM simulation.

Electrowetting on Dielectrics (EWOD) is a surface tension driven effect [1]. The shape of a fluid-gas interface, like a water droplet with a surrounding air phase, is strongly dependent on the surface tension equilibrium between the three governing phases of the considered system. Consequently, a FEM simulation of an EWOD process is an interface tracking process where surface tension and contact angles (CA) have to be known as exact as possible at every single position on the simulated fluid-gas interface to ensure a correct result of the whole EWOD simulation process. Two algorithms, namely the "LevelSet" and the "PhaseField" interface tracking algorithms are implemented in the used simulation software COMSOL ${ }^{\mathrm{TM}}$ Multiphysics 5.2 [2][3]. The used "PhaseField" interface tracking method coupled with the Navier-Stokes equations is one of the most promising and rather new approaches for handling moving contact lines and thin fluid-fluid interfaces [4]. Numerical mass conservation of the involved fluid phases is a main task to observe. The velocity of EWOD moved droplets and inertia induced oscillations of the interface during EWOD actuation strongly depend on the mass of the droplet. Therefore a change in mass due to numerical errors is harmful for the whole simulation effort. Also a dense mesh in the region of the triple contact line is required to resolve the contact angle as accurately as possible.

\section{Theoretical Background}

A sessile droplet resting on a surface has a contact angle determined by the equilibrium of the surface tensions $Y_{x y}$ between the three governing phases of the system (subscripts: $L$ liquid, S - solid, G - gas) (see Fig 1). The triple contact line is considered as the point or line 
where all these phases are in contact with each other. For a sessile droplet in three dimensions this is a circularly shaped baseline. The equilibrium and therefore the contact angle is described by Young's law [1]

$$
\gamma_{\mathrm{LG}} \cos \theta=\gamma_{\mathrm{SG}}-\gamma_{\mathrm{SL}}
$$

where $\theta$ is the resulting CA. In case of EWOD this equilibrium is modified by an extra voltage induced in (1). This extra term originates from surface charges gathering at the triple contact line yielding an extra force term in the Navier Stokes momentum equation, if a voltage is applied across the triple contact line (i.e. between the droplet and an underlying insulated electrode beneath). The resulting Lippmann Young formula reads as follows [5]

$$
\cos \theta_{\mathrm{V}}=\frac{\gamma_{\mathrm{SG}}-\gamma_{\mathrm{SL}}+\frac{\epsilon_{0} \epsilon_{\mathrm{r}} \mathrm{V}^{2}}{2 \mathrm{t}}}{\gamma_{\mathrm{LG}}}
$$

$\theta_{V}$ is the new CA under the influence of EWOD actuation, $Y_{X Y}$ is the surface tension between the considered phases, $\epsilon_{r}$ describes the dielectric material properties, $V$ is the applied EWOD voltage and $t$ is the combined thickness of the dielectric and hydrophobic layer. The setup for an EWOD experiment consists of an insulated electrode beyond the sessile droplet providing a well-defined electric potential. Often an extra hydrophobic layer is added to obtain a well-defined contact angle. The variable $t$ is the combined thickness of the dielectric insulation layer and the hydrophobic layer. In the examples treated in this work we assume an initial contact angle of $90^{\circ}$ allowing a simpler geometric modeling of the situation.

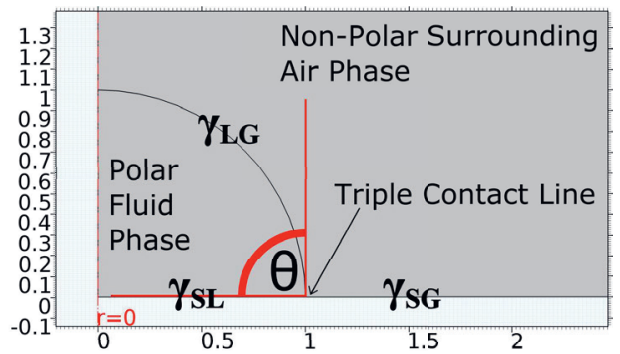

Fig. 1. Modeled $2 d$ axialsymmetric sample geometry for a sessile droplet on top of an EWOD stack with an initial $C A$ of $\theta_{0}=90^{\circ}$ and radius $r=1 \mathrm{~mm}$.

An EWOD channel, which is discussed in the example chapter 5 , consists of the electrode stack mentioned above and an extra stack in contact on top of the droplet to provide the electrical ground.

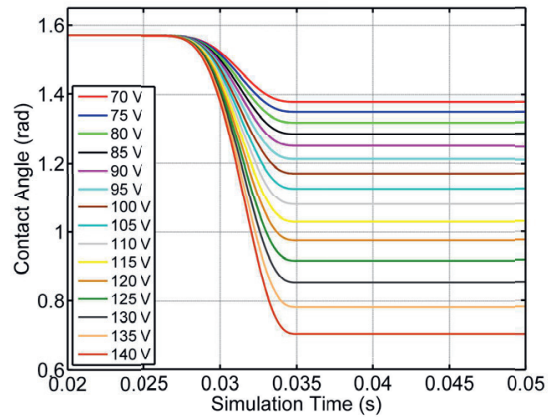

Fig. 2. The simulated change of a sessile droplet's contact angle (initial CA $=90^{\circ}$ or 1,5708 radiant) for various applied EWOD voltages beyond the droplet's triple contact line versus time.

The main question is now how to ensure the correct implementation of the boundary conditions and the used simulation algorithms. Fluid dynamic simulations numerically solve the Navier Stokes equations, which can behave rather chaotic on changing initial and boundary conditions. So the results should only be trusted after heavy observation and validation of the correct implications and conditions used for a specific problem. Of course an alignment and double checking with experimental data will always be the ultimate verification of the implemented model.

\section{Modeling of an EWOD CA Change Process}

The sessile droplet has been modeled as quarter circle with an initial CA of $\theta_{0}=90^{\circ}$, resting on a smooth, even surface (see Fig 1 ). The simulations are implemented as axial symmetric 2D FEM simulations where the $z$ axis (axis of symmetry) describes the height of the droplet and the $r$ axis is parallel to the surface where the droplet rests. The contact angle change has been modeled by applying a boundary condition to the wall region beyond the droplets triple contact line. The contact angle $\theta$ has been defined by applying the $\cos ^{-1}$ function appearing in the Lippmann Young formula. A voltage boundary condition has been applied to the same region. So if $V=0, \theta=\theta_{0}$ which is $90^{\circ}$ in all our simulations. For $V \neq 0$ the cosine of the contact angle equals the values shown in Fig. 2. after a short time for the droplet to adapt to the new CA. The following figures now show a difference in settling to the newly created CA equilibrium for the two used interface tracking algorithms. We particularly focus on the droplet's interface front velocity in $z$ direction.

Figs. 3-4 show the course of the interface front's z-velocity versus time for different dynamic viscosities $\eta$ simulated with the LevelSet interface tracking algorithm. At 
$t=0.3 \mathrm{~s}$ simulation time, the voltage is turned on and the observed values oscillate around their new equilibrium value. After applying a voltage, the droplet slowly settles to the new CA given by the Lippmann Young law. Inertia leads to an oscillation around the new state of equilibrium in the droplet's front velocity. The oscillation gets heavily damped if highly viscous fluids are considered.

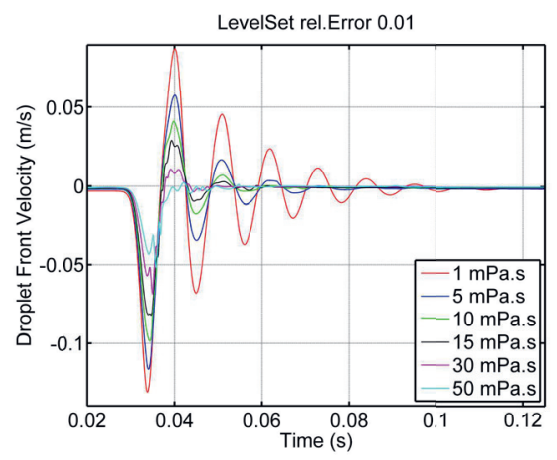

Fig. 3. Simulation of an EWOD actuation process using the LevelSet algorithm. Velocity of the droplet front during an EWOD actuation process versus time for various dynamic viscosities $\eta$.

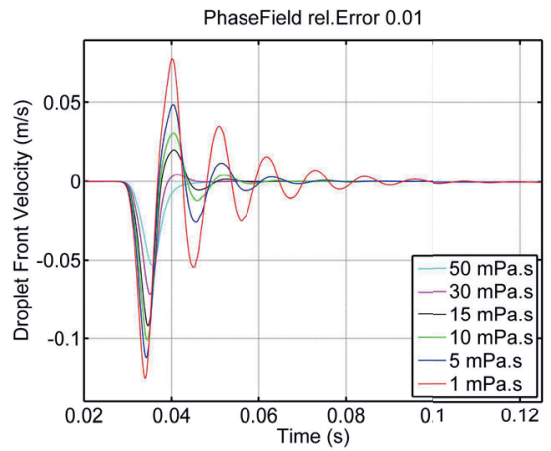

Fig. 4. Simulation of an EWOD actuation process using the Phase Field algorithm. Velocity of the droplet front during an EWOD actuation process versus time for various dynamic viscosities $\eta$.

The first observation is a superimposed oscillation in the droplets front velocity. These oscillations occur in the beginning of the simulation process where the change in front velocity is rather fast and seems to disappear at about $t=0.6 \mathrm{~s}$. The LevelSet algorithm is known to produce overshoots for fast changes in small variables, which results in a spurious oscillation of the solution at that point.

Fig. 4 shows the corresponding simulation using the PhaseField interface tracking algorithm. No superimposed oscillations can be seen in the droplet's front velocity.

The second observation is the larger oscillation amplitude of the LevelSet Method, compared to the PhaseField Method. A possible explanation for this behavior is the inferior numerical mass conservation of the LevelSet method. During simulation, numerical mass loss appears and leads to stronger oscillations, due to the reduced mass of the whole fluid droplet. In summary, the PhaseField method has to be preferred for this kind of EWOD FEM simulation. The more stable algorithm allows us to raise the relative error without spuriously affecting the solution, which leads to a lower computational effort. In addition, the achieved numerical mass conservation is better because of an extra PhaseField help variable $\varphi$ which is held constant by the solver and basically represents the mass of the simulated fluid. Therefore all following simulations are performed with the PhaseField interface tracking algorithm. The time step size has to be aligned with the mesh size for both simulation algorithms (CFL condition [6]) and has been set to $\Delta t=0.001 \mathrm{~s}$.

\section{Mesh Quality and Mass Conservation}

Fig. 5 shows the time course of the PhaseField auxiliary variable $\varphi$ (Water phase) for our test system, the sessile droplet on an even surface with a CA of $90^{\circ}$ and a radius of $r=1 \mathrm{~mm}$. The variable maxEl denotes to the distance between two mesh nodes in $\mathrm{mm}$. A smaller maxEl corresponds to a finer mesh. For a very coarse mesh, we observe heavy numerical mass loss of the water phase. The reasons are calculation errors during the geometric reconstruction process between every time step. These errors become smaller with a higher mesh density. That can be seen for higher mesh qualities ( $\operatorname{maxEl}=0.0125 \mathrm{~mm}$ and beyond). There is virtually no visible difference in $\varphi$ between $\max E I=0.0125 \mathrm{~mm}$ and $\max E I=0.01 \mathrm{~mm}$. A further improvement of the mesh density thus does not enhance the mass conservation behavior anymore.

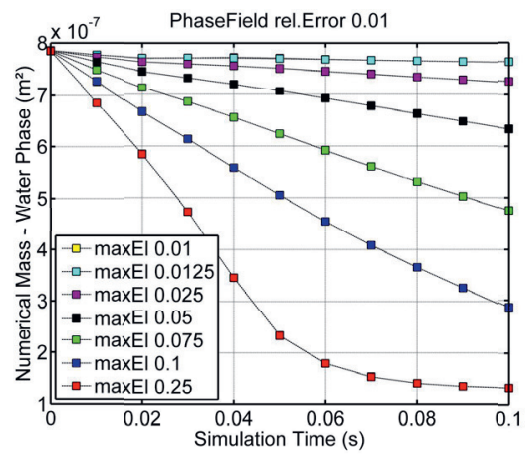

Fig. 5. Phase field variable $\varphi$ (essentially the numerical mass) versus a simulation time of $0.1 \mathrm{~s}$ for a sessile droplet resting above an EWOD electrode for increasing mesh quality. The slope of the mass loss of the water phase reduces as the maximum mesh element size decreases [7]. 
A mesh convergence analysis, like the one shown in Fig. 6 is a standard procedure during setting up a Computational Fluid Dynamics (CFD) simulation. Like already assumed, the mass of the system has of course heavy impact on the solution behavior.

Dense meshes require a high computional effort. Therefore, the mesh should be adapted and refined only in the region were a dense mesh is needed. In interface tracking simulations this is of course the interface region between the fluid phases. All simulations in this work were performed with an adapting mesh refinement algorithm to save computational time. The proper mesh design also ensures that the observed superimposed oscillations from chapter 3 are not an effect of an inadequate mesh density.

\section{Example: Scaling a Microchannel for EWOD Display Applications}

In recent works we presented an EWOD microchannel, where a droplet is actuated forth and back to represent the switched on and off state of a microfluidic pixel [8]. The main focus was to test the scalability of these pixels for applications from small smartphone displays to large displays, e.g., advertisement or traffic signs. Fig. 6 shows the scaling limit and wetting diameter of a droplet in contact with both sides of an EWOD channel. The channel is scaled up until the surface tension forces cannot balance the gravitational forces anymore. Then, the fluid loses the contact to the top GND electrode and spreads inside the channel, indicating the scaling limit of this microfluidic pixel channel.

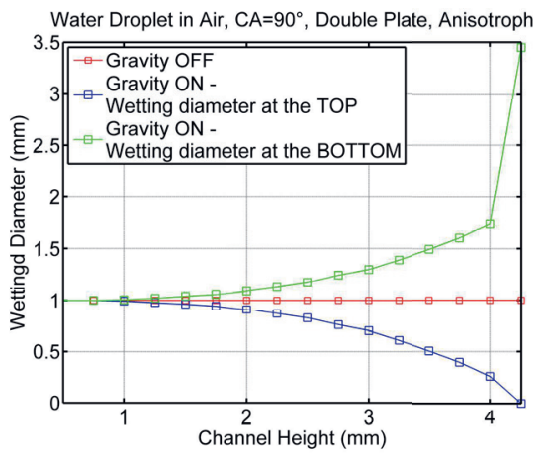

Fig. 6. Droplet inside an upscaled fluidic channel (growth only in y direction - anisotropic scaling).

If we increase the distance between the bottom and the top EWOD stack (anisotropic upscaling only in $y$ direction of a 2D FEM Channel simulation), the contact to the top GND electrode is lost at a channel height of about $4 \mathrm{~mm}$.

\section{Conclusion}

This work demonstrates the benefits of the PhaseField algorithm for simulating EWOD induced CA changes. The instability of the LevelSet method against fast changes in certain variables leads to spurious oscillations of the solution while the PhaseField method shows none of these inaccuracies. The advantage of the PhaseField method in terms of mass conservation lies within its extra PhaseField auxiliary conservation variable $\varphi$. With this knowledge the scaling behavior of a fluid channel used for microfluidic display applications has been examined using the PhaseField algorithm. These simulations show the anisotroph scaling limits of such a display channel with a single droplet actuated by EWOD forces inside and show the maximum channel height, until the surface tension is not strong enough to balance the gravitational force. This cuts the contact between the droplet and the GND electrode, corrupting the functionality of the fluidic pixel.

\section{Acknowledgment}

This work has been supported by the Austrian Research Promotion Agency FFG (843829)

\section{References}

[1] M. Vallet, B. Berge, L. Vovelle, Electrowetting of water and aqueous solutions on poly(ethylene terephthalate) insulating films, Polymer (Guildf). 37 (1996) 2465-2470. doi:10.1016/00323861(96)85360-2.

[2] D. Jacqmin, Calculation of Two-Phase NavierStokes Flows Using Phase-Field Modeling, J. Comput. Phys. 155 (1999) 96-127. doi:10.1006/jcph.1999.6332.

[3] E. Olsson, G. Kreiss, A conservative level set method for two phase flow, J. Comput. Phys. 210 (2005) 225-246. doi:10.1016/j.jcp.2005.04.007.

[4] X. Cai, H. Marschall, M. Wörner, O. Deutschmann, Numerical Simulation of Wetting Phenomena with a Phase-Field Method Using OpenFOAM, Chem. Eng. Technol. 38 (2015) 1985-1992. doi:10.1002/ceat.201500089.

[5] G. Lippmann, Relations entre les phenomenes electriques et capillaries, Comptes Rendus l'Académie Des Sci. (1875).

[6] K.N. Chaudhury, K.R. Ramakrishnan, Stability and convergence of the level set method in computer vision, Pattern Recognit. Lett. 28 (2007) 884-893. doi:10.1016/j.patrec.2006.12.003.

[7] A. Tröls, H. Enser, S. Clara, B. Jakoby, FEM Simulation and Experimental Realization of an Optical Readout Method for a Low-Cost Screen Printed Optofluidic Switch Based on Electrowetting on Dielectrics (EWOD) Forces, Unpubl. Submitt. to Sensors Actuators A. (2017).

[8] A. Tröls, H. Enser, B. Jakoby, Modeling and Fabrication of Low-Cost Electrowetting Actuators for Flexible Microfluidic Display Applications, in: 2016 IEEE SENSORS - Proc., 2016: pp. 94-96. doi:10.1109/ICSENS.2016.7808429. 